\title{
Towards a molecular classification of pulmonary arterial hypertension
}

\author{
Stefan Gräf and Nicholas W. Morrell \\ Affiliation: Dept of Medicine, University of Cambridge School of Clinical Medicine, Cambridge, UK. \\ Correspondence: Nicholas W. Morrell, University of Cambridge, Dept of Medicine, Box 157, Level 5, \\ Addenbrooke's Hospital, Hills Road, Cambridge, CB2 2QQ, UK. E-mail: nwm23Acam.ac.uk
}

@ERSpublications

This editorial discusses recent advances in understanding the genetic basis of pulmonary arterial hypertension http://ow.ly/mmfi303fvCu

Pulmonary arterial hypertension $(\mathrm{PAH})$, although rare, remains a serious and life-limiting condition despite the availability of existing therapies [1]. In particular, the diagnosis of idiopathic PAH (in the absence of known disease gene associations) carries a 3 year survival of $60-70 \%$ in most registries [2]. The survival is even poorer for patients with familial $\mathrm{PAH}$, who have at least one affected relative [3]. It is likely that the key to finding better treatments for patients with rare and severe forms of PAH is an improved understanding of the cellular and molecular basis of this disease, since knowledge of the genetics provides important clues to the major pathways to be targeted. For many rare diseases, rare high impact mutations in single genes play a major role in their pathobiology. Indeed, we now know that in families with $\mathrm{PAH}$ over $80 \%$ of cases are caused by heterozygous germline mutations in the gene (BMPR2) encoding the bone morphogenetic protein type II receptor (BMPR-II), a receptor for the transforming growth factor- $\beta$ family of ligands [3]. In sporadic cases of idiopathic PAH, mutations in BMPR2 are found in $15-20 \%$ of cases [3]. These latter cases appear to be sporadic, rather than familial, because the mutations in BMPR2 exhibit low penetrance (less than $50 \%$ of carriers are affected, with females more susceptible than males), while some sporadic cases present with de novo mutations in the gene [4]. The presence of a known causal mutation in a PAH gene is classified as heritable PAH. Understanding the role played by BMPR-II in PAH pathobiology has led to several preclinical approaches to treating this disease [5-7] that are now being translated into experimental medicine studies in patients [8].

Although mutations in BMPR2 are the commonest genetic cause of PAH, they do not explain all cases, especially the sporadic cases. Over the past 15 years mutations have been identified in additional genes (reviewed in [9]). Interestingly, several of these are within the bone morphogenetic protein (BMP) and transforming growth factor- $\beta$ signalling pathways, for example ACVRL1 (encoding activin-receptor-like kinase 1 (ALK1)) [10] and ENG (encoding endoglin) [11]. Mutations in these genes are usually found in families with hereditary haemorrhagic telangiectasia (HHT), but occasionally these individuals present with severe PAH. The link appears to be that both ALK1 and endoglin are essential for BMP signalling in pulmonary artery endothelial cells. In addition, mutations in the genes encoding the downstream signalling intermediaries for BMP receptors, the Smad proteins (particularly SMAD1 and SMAD9) have been reported in some patients [9]. Further mutations have been reported in genes not immediately associated with BMP signalling, including CAV1 (encoding caveolin-1) [12] and KCNK3 (encoding a potassium channel) [13]. There have also been reports of autosomal dominant mutations in the gene TBX4 in patients with PAH, usually in families affected by the small patella syndrome [14]. However, taken together, mutations in non-BMPR2 genes account for less than 5\% of PAH cases, at least in mainly adult cohorts. In terms of understanding the pathobiology, the low frequency of mutations in these genes does not make them any less important in $\mathrm{PAH}$, since they all represent vital clues in defining new therapeutic targets. The challenge will be in working out how and whether these pathways interact to drive the pathology of pulmonary vascular remodelling, and where the "sweet spots" are for intervention.

Received: Aug 032016 | Accepted after revision: Aug 112016

Conflict of interest: None declared.

Copyright OERS 2016 
More recently, the genetic basis of an ultra-rare form of PAH, pulmonary veno-occlusive disease (PVOD) or pulmonary capillary haemangiomatosis, was shown (especially in families with PVOD) to be largely due to recessive biallelic mutations in the gene EIF2AK4, which encodes a kinase for the integrated stress response. As with other recessive rare conditions, consanguinity is more common in the parents who are carriers for the EIF2AK4 mutation [15-17]. These interesting new findings were recently reviewed in detail [18].

It might be anticipated that diseases driven by Mendelian genetic mutations would occur at a younger age and potentially be more severe. This has been confirmed recently in PAH patients with mutations in BMPR2 [3]. The corollary is that one might expect the burden of genetic causes of PAH to be higher in children with the disease. The report by Levy et al. [19] in this issue of the European Respiratory Journal addresses this by sequencing the genes identified to date that are thought to be causal in PAH. In total, 66 cases of paediatric PAH were sequenced for the known genes. Of these, 23 children had PAH associated with coincidental congenital heart disease or following repair of cardiac defects. No pathogenic mutations in the known genes were found in this group. In a further 40 children with idiopathic or familial PAH, the investigators found 12 pathogenic mutations (30\% of this group) in the known genes. Mutations in BMPR2 were found in $12.5 \%$ of cases, $A C V R L 1$ in $10 \%$ and TBX4 in $7.5 \%$. No mutations were found in KCNK3 or SMAD9. Three patients had been clinically diagnosed with PVOD, of which two were found to carry biallelic mutations in EIF2AK4. In general, patients with causal PAH mutations had more severe disease at diagnosis and were likely to receive more aggressive first line therapy. The authors were unable to ascertain the impact of mutations in individual genes on clinical outcomes probably because of the small numbers studied, despite being recruited from a national referral centre over a period of 6 years. This highlights one of the major problems with studying the genetics of a rare disease even at a national level. To fully address the impact of genetics on clinical phenotype and disease natural history will require close international collaboration between centres, especially in the field of paediatric PAH.

The authors claim that paediatric PAH has a specific genetic architecture. The evidence for this was the finding that non-BMPR2 mutations are more common in this cohort of children than previously reported in adult populations with idiopathic and familial PAH. This is interesting and might indeed be the case, but further studies are required to ascertain the true prevalence in larger paediatric populations which can take account of local referral patterns or expertise. The authors recognise that prevalence of mutations may differ in incident and prevalent cohorts of patients, with the prevalent group representing those more likely to survive $[20,21]$. Most striking was the prevalence of mutations in TBX4 and ACVRL1. In these cases there should be a family history of small patella syndrome [22] or HHT, respectively, highlighting the importance of taking a careful family history in children with PAH. Of note, although highly penetrant, HHT may not manifest clinically (e.g. epistaxis) until adolescence. Small patella syndrome may be suggested by the clinical findings of small or absent patella, and a wide space between the first and second toes [22]. In a previously reported Dutch cohort of 20 paediatric idiopathic and familial $\mathrm{PAH}$ cases [14], TBX4 mutations, accompanied by clinical signs of small patella syndrome, accounted for $30 \%$ of the patients, but again local referral patterns might have led to over-representation of this diagnosis. It is intriguing that TBX4 mutations seem to account for a very small proportion of PAH in adults and yet TBX4 mutations in childhood do not appear to cause more severe disease, making it unlikely that early death is the cause of the lower frequency in adults. It should also be noted that the frequency of $\mathrm{PAH}$ in cohorts of patients with small patella syndrome is very low, suggesting low penetrance of the $\mathrm{PAH}$ phenotype.

Nevertheless, TBX4 is now firmly validated as a causal gene in $\mathrm{PAH}$, that is potentially enriched in paediatric cases. The TBX4 transcription factor is involved in the regulation of BMP signalling, but is largely underexplored in this context outside of embryonic development [23]. Much might be learned from a more thorough evaluation of the role of this gene in PAH.

It is becoming clear that the diagnostic entity of idiopathic $\mathrm{PAH}$ in children or in adults has heterogeneous underlying genetic causes. Large-scale international collaborative efforts are required to elucidate the true clinical utility of genetic information in PAH with regard to risks to family members, clinical course, response to therapy and the need for early aggressive therapy. At the present time our management of patients is largely guided by haemodynamic findings at right heart catheterisation. It is possible that in the future such decisions might be aided by the underlying genetic diagnosis, for which highly specific therapies might become available, as is the case, for example, in cystic fibrosis. Improved diagnostic genetic testing platforms deploying targeted sequencing of gene panels will enable us to assess several disease genes in parallel to unveil a more comprehensive view of the more complex genetic architectures underlying the disease.

The rapid advances in understanding of the genetics of PAH means that clinicians caring for patients with this condition will need to be more prepared to discuss the implications for patients and their families, and to consider the need for diagnostic testing in patients and predictive testing in family members [24]. 
Much remains to be discovered about the genetics of PAH. It is highly likely that rare causal mutations in novel genes account for a proportion of additional cases of PAH. International efforts are underway and are likely to report initial findings within the next year. The influence of more common genetic variation and the interaction with the environment, perhaps through epigenetic mechanisms, remains to be explored, but a thorough knowledge of the underlying high impact mutations is an important first step. As part of this process it is important that we curate the truly causal high impact mutations in this disease [25-27]. The rapid advances in whole exome and whole genome sequencing technology means that we need to be vigilant to ensure that genetic findings can be replicated and validated in cohorts of sufficient size, and ensure that potentially causal variants are carefully assessed to avoid misleading ourselves and our patients [28-30].

\section{References}

1 Galiè N, Humbert M, Vachiery JL, et al. 2015 ESC/ERS Guidelines for the diagnosis and treatment of pulmonary hypertension. Eur Respir J 2015; 46: 903-975.

2 Farber HW, Miller DP, Poms AD, et al. Five-year outcomes of patients enrolled in the REVEAL Registry. Chest 2015; 148: 1043-1054.

3 Evans JD, Girerd B, Montani D, et al. BMPR2 mutations and survival in pulmonary arterial hypertension: an individual participant data meta-analysis. Lancet Respir Med 2016; 4: 129-137.

4 Austin ED, Loyd JE. The genetics of pulmonary arterial hypertension. Circ Res 2014; 115: 189-202.

5 Morrell NW, Bloch DB, ten Dijke P, et al. Targeting BMP signalling in cardiovascular disease and anaemia. Nat Rev Cardiol 2016; 13: 106-120.

6 Long L, Ormiston ML, Yang X, et al. Selective enhancement of endothelial BMPR-II with BMP9 reverses pulmonary arterial hypertension. Nat Med 2015; 21: 777-785.

7 Spiekerkoetter E, Tian X, Cai J, et al. FK506 activates BMPR2, rescues endothelial dysfunction, and reverses pulmonary hypertension. J Clin Invest 2013; 123: 3600-3613.

8 Spiekerkoetter E, Sung YK, Sudheendra D, et al. Low-dose FK506 (tacrolimus) in end-stage pulmonary arterial hypertension. Am J Respir Crit Care Med 2015; 192: 254-257.

9 Machado RD, Southgate L, Eichstaedt CA, et al. Pulmonary arterial hypertension: a current perspective on established and emerging molecular genetic defects. Hum Mutat 2015; 36: 1113-1127.

10 Trembath RC, Thomson JR, Machado RD, et al. Clinical and molecular genetic features of pulmonary hypertension in patients with hereditary hemorrhagic telangiectasia. N Engl J Med 2001; 345: 325-334.

11 Chaouat A, Coulet F, Favre C, et al. Endoglin germline mutation in a patient with hereditary haemorrhagic telangiectasia and dexfenfluramine associated pulmonary arterial hypertension. Thorax 2004; 59: 446-448.

12 Austin ED, Ma L, LeDuc C, et al. Whole exome sequencing to identify a novel gene (caveolin-1) associated with human pulmonary arterial hypertension. Circ Cardiovasc Genet 2012; 5: 336-343.

13 Ma L, Roman-Campos D, Austin ED, et al. A novel channelopathy in pulmonary arterial hypertension. $N$ Engl J Med 2013; 369: 351-361.

14 Kerstjens-Frederikse WS, Bongers EM, Roofthooft MT, et al. TBX4 mutations (small patella syndrome) are associated with childhood-onset pulmonary arterial hypertension. J Med Genet 2013; 50: 500-506.

15 Eyries M, Montani D, Girerd B, et al. EIF2AK4 mutations cause pulmonary veno-occlusive disease, a recessive form of pulmonary hypertension. Nat Genet 2014; 46: 65-69.

16 Best DH, Sumner KL, Austin ED, et al. EIF2AK4 mutations in pulmonary capillary hemangiomatosis. Chest 2014 145: 231-236.

17 Tenorio J, Navas P, Barrios E, et al. A founder EIF2AK4 mutation causes an aggressive form of pulmonary arterial hypertension in Iberian Gypsies. Clin Genet 2015; 88: 579-583.

18 Montani D, Lau EM, Dorfmüller P, et al. Pulmonary veno-occlusive disease. Eur Respir J 2016; 47: 1518-1534.

19 Levy M, Eyries M, Szezepanski I, et al. Genetic analyses in a cohort of children with pulmonary hypertension. Eur Respir J 2016; 48: 1118-1126.

20 Humbert M, Sitbon O, Yaïci A, et al. Survival in incident and prevalent cohorts of patients with pulmonary arterial hypertension. Eur Respir J 2010; 36: 549-555.

21 Simonneau G, Channick RN, Delcroix M, et al. Incident and prevalent cohorts with pulmonary arterial hypertension: insight from SERAPHIN. Eur Respir J 2015; 46: 1711-1720.

22 Bongers EM, Duijf PH, van Beersum SE, et al. Mutations in the human TBX4 gene cause small patella syndrome. Am J Hum Genet 2004; 74: 1239-1248.

23 Rodriguez-Esteban C, Tsukui T, Yonei S, et al. The T-box genes Tbx4 and Tbx5 regulate limb outgrowth and identity. Nature 1999; 398: 814-818.

24 Girerd B, Montani D, Jaïs X, et al. Genetic counselling in a national referral centre for pulmonary hypertension. Eur Respir J 2016; 47: 541-552.

25 Kircher M, Witten DM, Jain P, et al. A general framework for estimating the relative pathogenicity of human genetic variants. Nat Genet 2014; 46: 310-315.

26 Minikel EV, Vallabh SM, Lek M, et al. Quantifying prion disease penetrance using large population control cohorts. Sci Transl Med 2016; 8: 322ra9.

27 Walsh R, Thomson K, Ware JS, et al. Reassessment of Mendelian gene pathogenicity using 7,855 cardiomyopathy cases and 60,706 reference samples. Genet Med 2016; in press [DOI: http://dx.doi.org/10.1101/041111].

28 Walter K, Min JL, Huang J, et al. The UK10K project identifies rare variants in health and disease. Nature 2015 526: 82-90.

29 Exome Aggregation Consortium, Lek M, Karczewski K, et al. Analysis of protein-coding genetic variation in 60,706 humans. Nature 2016; in press [DOI: 10.1101/030338].

30 Telenti A, Pierce LT, Biggs WH, et al. Deep sequencing of 10,000 human genomes. bioRxiv 061663; http://dx.doi. org/10.1101/061663 